\title{
New Conditions for the Exponential Stability of Pseudolinear Difference Equations in Banach Spaces
}

\author{
Rigoberto Medina \\ Departamento de Ciencias Exactas, Universidad de Los Lagos, Casilla 933, 5290000 Osorno, Chile \\ Correspondence should be addressed to Rigoberto Medina; rmedina@ulagos.cl
}

Received 28 December 2015; Revised 18 March 2016; Accepted 24 March 2016

Academic Editor: Patricia J. Y. Wong

Copyright (C) 2016 Rigoberto Medina. This is an open access article distributed under the Creative Commons Attribution License, which permits unrestricted use, distribution, and reproduction in any medium, provided the original work is properly cited.

\begin{abstract}
We study the local exponential stability of evolution difference systems with slowly varying coefficients and nonlinear perturbations. We establish the robustness of the exponential stability in infinite-dimensional Banach spaces, in the sense that the exponential stability for a given pseudolinear equation persists under sufficiently small perturbations. The main methodology is based on a combined use of new norm estimates for operator-valued functions with the "freezing" method.
\end{abstract}

\section{Introduction}

The problem of stability and robustness of difference systems has been extensively studied in the last years [1-7]. However, mainly systems with linear leading parts were investigated. In fact, a few investigations have dealt with stability conditions for nonlinear difference or differential systems (see, e.g., $[3,8-$ 14]). This itself highlights the importance of establishing new criteria to study exponential stability of nonlinear systems. Pseudolinear systems are an important class of nonlinear systems. The stability and robustness of pseudolinear differential equations are considered, for example, in [15-19].

Martynyuk [19] derived new bounds for solutions of perturbed pseudolinear differential equations, basically using Gronwall-type inequalities. Dvirnyi and Slyn'ko [16, 17], constructing a piecewise differential Lyapunov function, established the stability of solutions to impulsive differential equations with impulsive action in the pseudolinear form. Banks et al. [15], using a Gronwall-type inequality and assuming that a matrix $B(x, t)$ satisfies a jointly Lipchitz inequality in $x$ and $t$, established the robust exponential stability of evolution differential equations of pseudolinear form. In summary, in the existing literature there are many results concerning the stability or asymptotic behavior of pseudolinear differential equations; however, in general, the assumptions are difficult to check or conservative.
Our results compare favorably with the abovementioned works in the following sense:

(a) We established a local stability theory of discrete evolution equations.

(b) The Lipschitz assumptions are local in the state and general in the time.

(c) Explicit estimates to the norm of the associated evolution semigroups are established.

In this paper we consider evolution difference systems defined in infinite-dimensional Banach spaces, with bounded operators on the right-hand side represented in the pseudolinear form. New estimates for the norms of solutions are derived giving us explicit stability and boundedness conditions. Our approach is based on the generalization of the freezing method to abstract difference systems. The equations will be represented as a perturbation about a fixed value of the coefficient operator. Thus, applying norm estimates for the involved operator-valued functions, new stability results are established.

Although the freezing method appears to be often utilized in practice in the control of linear time-varying systems, not much is currently known regarding the stability or asymptotic behavior of pseudolinear difference systems of the form considered here. In fact, we will develop a local stability theory to evolution pseudolinear difference systems. 
The remainder of this paper is organized as follows: In Section 2, we introduce some notations, the statement of the problem, and a fundamental result concerning the exponential stability of abstract difference equations. In Section 3, sufficient conditions for the exponential stability of pseudolinear difference systems are given. In Section 4 pseudolinear difference systems in Hilbert spaces are studied. Finally, Section 5 is devoted to the discussion of our results: We highlight the main conclusions.

\section{Statement of the Problem}

Let $X$ be a Banach space with an arbitrary norm $\|\cdot\|, \rho(A)$ denotes the spectral radius of an operator $A$, and

$$
\Omega(R)=\{x \in X:\|x\| \leq R\}, \quad R \leq \infty .
$$

Consider in a Banach space $X$ the equation

$$
\Delta x(k)=B(x(k), k) x(k) ; \quad x(0)=x_{0}, k=0,1, \ldots,
$$

where $\Delta$ is the difference operator defined by $\Delta u(k)=u(k+$ $1)-u(k)$ and $B(z, k)$ are bounded linear operators in $X$, continuously depending on $z \in \Omega(R)$; that is,

$$
B(z, k): D(B(z, k)) \subseteq X \longrightarrow X
$$

is a linear bounded operator in $X$, for each $z \in X, k \geq 0$. $\Omega(R)$ :

Denote by $\omega_{\infty}(R)$ the set of sequences with values in

$$
\omega_{\infty}(R)=\{h(j) \in \Omega(R): j=0,1, \ldots\} .
$$

Additionally, assume that $B(z, k)$ satisfies the conditions

$$
\begin{aligned}
\left\|\left(B\left(h_{1}, k\right)-B(h, k)\right) \omega\right\| & \multicolumn{1}{c}{(R)\left\|h_{1}-h\right\|\|\omega\|,} \\
\|(B(h, k)-B(h, s)) \omega\| & \mu(R)|k-s|\|\omega\|, \\
& \left(h_{1}, h, \omega \in \Omega(R) ; k, s \geq 0\right),
\end{aligned}
$$

where $\gamma(R)$ and $\mu(R)$ are nonnegative constants independent of $k, s$, and $\omega$.

Remark 1. Here we will consider system (2) as a perturbation of a fixed operator $B\left(x_{0}, k_{0}\right)$. Thus, our results can be applied to robust stability; in fact, we can regard

$$
\Delta x(k)=B\left(x_{0}, k_{0}\right) x(k)
$$

as the nominal system and

$$
\Delta x(k)=B(x(k), k) x(k)
$$

as a system with state-dependent parametric perturbations.

In order to establish the stability properties of (2), we will formulate a fundamental theorem concerning the exponential stability of the following nonlinear system of difference equations.

Consider in $X$ the equation

$$
x(k+1)=A(k) x(k)+f(x(k), k) ; \quad k=0,1, \ldots,
$$

where $\{A(k)\}_{k=0}^{\infty}$ is a family of linear bounded operators in $X$ with a common domain $D(A(k)):=D \subseteq X$ and $f(\cdot, k)$ : $\Omega(R) \rightarrow X$ is continuous for each $k \geq 0$. In addition, there are nonnegative constants $\mu=\mu(R)$ and $q=q(R)$, such that

$$
\begin{aligned}
& \|f(z, k)\| \leq \mu\|z\|, \\
& \qquad(z \in \Omega(R) ; k=0,1, \ldots),
\end{aligned}
$$$$
\|(A(k)-A(s)) \omega\| \leq q|k-s|\|\omega\|,
$$

$(k, s \geq 0 ; \omega \in \Omega(R))$,

with $q$ inpendent of $k, s$, and $\omega$.

Definition 2. The zero solution of system (9) is exponentially stable with respect to a ball $\Omega(\eta)$ if there are constants $M>0$ and $c_{0} \in(0,1)$ such that any solution $x(k)$ of $(9)$ with initial condition $x(0)=x_{0} \in \Omega(\eta)$ satisfies the inequality

$$
\left\|x\left(k, x_{0}\right)\right\| \leq M c_{0}^{k}\left\|x_{0}\right\|, \quad \forall k=0,1, \ldots
$$

Denote by $\Psi=\left\{A^{k}(j)\right\}_{k \geq j \geq 0}$ the discrete evolution family generated by the operator $A(j)$, for $j \geq 0$.

Theorem 3 (see [20]). Under conditions (10), assume that

$$
\begin{gathered}
M=\sup _{k \geq 0}\left(\sup _{l \geq 0}\left\|A^{k}(l)\right\|\right)<\infty, \\
\theta_{0}(R)=\sum_{k=0}^{\infty}(q k+\mu) \sup _{l \geq 0}\left\|A^{k}(l)\right\|<1 .
\end{gathered}
$$

Then the zero solution of (9) is exponentially stable with respect to a ball $\Omega(R)$. Moreover, any solution of (9) with initial vector $x_{0} \in \Omega(R)$ satisfies the inequality

$$
\sup _{k \geq 1}\|x(k)\| \leq \frac{M\left\|x_{0}\right\|}{1-\theta_{0}},
$$

provided that

$$
M\left\|x_{0}\right\|\left(1-\theta_{0}\right)^{-1}<R
$$

\section{Main Results}

Now, returning to system (2), we write it in the form

$$
\begin{aligned}
\Delta x(k)= & B(h(s), s) x(k) \\
& +[B(x(k), k)-B(h(s), s)] x(k), \\
& k=0,1, \ldots,
\end{aligned}
$$

with a fixed integer $s$.

Put

$$
\begin{aligned}
& M(R)=\sup _{h \in \Omega(R), k \geq 0}\|B(h, k) h\|, \\
& q_{0}(R)=\gamma(R) M(R)+\mu(R) .
\end{aligned}
$$


Assume that

$$
p(R, k)=\sup _{h \in \Omega(R), s \geq 0}\left\|\widehat{B}^{k}(h, s)\right\|<\infty,
$$

where $\widehat{B}(h, k)=I+B(h, k)$, with $I$ the identity operator, and

$$
\chi(R):=\sup _{k \geq 0} p(R, k)<\infty .
$$

Theorem 4. For a positive $R<\infty$, let conditions (5), (6), (16)(18), and

$$
\widetilde{\theta}_{0}(R)=q_{0}(R) \sum_{k=0}^{\infty} k p(R, k)<1
$$

hold. Then the zero solution of (2) is exponentially stable. Moreover, the solution $x(k)$ of (2), with initial condition $x_{0} \in$ $\Omega(R)$, is subject to the estimate

$$
\sup _{k \geq 1}\|x(k)\| \leq \chi(R)\left\|x_{0}\right\|\left(1-\widetilde{\theta}_{0}(R)\right)^{-1},
$$

provided that

$$
\chi(R)\left\|x_{0}\right\|\left(1-\widetilde{\theta}_{0}(R)\right)^{-1}<R .
$$

Proof. Let us introduce the equation

$$
\Delta y_{h}(k)=B(h(k), k) y_{h}(k), \quad k=0,1, \ldots,
$$

where $h \in \omega_{\infty}(R)$. If $h(k)=x(k)$ is a solution of (2), then (22) and (2) coincide. If we put $A(k)=B(h(k), k)$, with the function $h(k)$, then (22) takes the form

$$
\Delta x(k)=A(k) x(k), \quad k=0,1, \ldots
$$

By (5) and (6) we have

$$
\begin{aligned}
&\|B(h(k), k)-B(h(s), s)\| \\
& \leq\|B(h(k), k)-B(h(s), k)\| \\
&+\|B(h(s), k)-B(h(s), s)\| .
\end{aligned}
$$

On the other hand, by (16), we have $\|\Delta x(k)\| \leq M(R)$, so it follows that $\|x(k)-x(s)\| \leq M(R)|k-s|, \forall k \geq s \geq 0$.

Consequently

$$
\begin{aligned}
\|B(h(k), k)-B(h(s), s)\| & \\
\quad \leq \gamma(R)\|x(k)-x(s)\|+\mu(R)|k-s| & \\
\leq(\gamma(R) M(R)+\mu(R))|k-s|=q_{0}(R)|k-s|, & \forall k \geq s \geq 0 .
\end{aligned}
$$

Thus, applying Theorem 3 to (23) with $A(k)=\widehat{B}(h(k), k)$ and $f \equiv 0$, the result follows.

Remark 5. Theorem 4 remains true if the linear system

$$
\Delta x(k)=B(0, k) x(k), \quad k=0,1, \ldots
$$

satisfies conditions (17) and (18), and in this case condition (19) takes the form

$$
\tilde{\theta}_{0}(R)=\mu(R) \sum_{k=0}^{\infty} k p(R, k)<1 .
$$

\section{Pseudolinear Systems in Hilbert Spaces}

As the previous theorems show, the extension of the freezing method to evolution equations is based on norm estimates for relevant semigroups. However, obtaining these estimates is usually not an easy task. Because of this, we restrict ourselves by equations in a separable Hilbert space with HilbertSchmidt coefficient operators.

In the finite-dimensional case, the spectrum of a linear operator consists of its eigenvalues. The spectral theory of bounded linear operators on infinite-dimensional spaces is an important but challenging area of research. For example, an operator may have a continuous spectrum in addition to, or instead of, a point spectrum of eigenvalues. A particularly simple and important case is that of compact, self-adjoint operators. Compact operators may be approximated by finitedimensional operators, and their spectral theory is close to that of finite-dimensional operators. We will assume that coefficient operators of (2) are not necessarily compact or selfadjoint. See, for example, [21, 22].

To formulate the next results, let us introduce the following notations and definitions: Let $H$ be a separable Hilbert space and $A$ a linear compact operator in $H$. If $\left\{e_{k}\right\}_{k=1}^{\infty}$ is an orthogonal basis in $H$ and the series $\sum_{k=1}^{\infty}\left(A e_{k}, e_{k}\right)$ converges, then the sum of the series is called the trace of the operator $A$ and is denoted by

$$
\operatorname{Trace}(A)=\operatorname{Tr}(A)=\sum_{k=1}^{\infty}\left(A e_{k}, e_{k}\right)
$$

Definition 6. An operator $A$ satisfying the relation

$$
\operatorname{Tr}\left(A^{*} A\right)<\infty
$$

is said to be a Hilbert-Schmidt operator, where $A^{*}$ is the adjoint operator of $A$.

The norm

$$
N_{2}(A)=N(A)=\sqrt{\operatorname{Tr}\left(A^{*} A\right)}
$$

is called the Hilbert-Schmidt norm of $A$.

Definition 7. A bounded linear operator $A$ is said to be quasiHermitian if its imaginary component

$$
A_{I}=\frac{A-A^{*}}{2 i}
$$

is a Hilbert-Schmidt operator, where $A^{*}$ is the adjoint operator of $A$.

Theorem 8 (see [22], p. 118). Let $A$ be a bounded linear operator acting on a separable Hilbert space and satisfying (31). Then

$$
\left\|A^{m}\right\| \leq \sum_{k=0}^{m} \frac{m !(\rho(A))^{m-k} g_{I}^{k}(A)}{(m-k) !(k !)^{3 / 2}}
$$

for any integer $m \geq 1$, where $g_{I}(A)=\sqrt{2}\left[N_{2}^{2}\left(A_{I}\right)-\right.$ $\left.\sum_{k=1}^{\infty}\left|\operatorname{Im} \lambda_{k}(A)\right|^{2}\right]^{1 / 2}$, and $\lambda_{k}(A)$ are the eigenvalues of $A$, 
including their multiplicities, and $\rho(A)$ is the spectral radius of A. Assume that

$$
\begin{aligned}
& \widehat{v}_{0}=\sup _{k \geq 0} g_{I}(A(k))<\infty, \\
& \hat{\rho}_{0}=\sup _{k \geq 0} \rho(A(k))<1 .
\end{aligned}
$$

Denote

$$
\widehat{M}_{0}=\sup _{m \geq 1}\left(\sum_{k=0}^{m} \frac{m ! \widehat{\rho}_{0}^{m-k} \widehat{v}_{0}^{k}}{(m-k) !(k !)^{3 / 2}}\right) .
$$

Theorem 9 (see [23]). Under conditions (10) and (33), assume that

(i) $A(k)(k=0,1, \ldots)$ are Hilbert-Schmidt operators satisfying condition (31),

(ii)

$$
\begin{aligned}
S(A(\cdot), f) & =\sum_{j=0}^{\infty} \frac{\widehat{v}_{0}^{j}}{\sqrt{j !}}\left[\frac{(j+1) q}{\left(1-\hat{\rho}_{0}\right)^{j+2}}+\frac{\mu}{\left(1-\widehat{\rho}_{0}\right)^{j+1}}\right] \\
& <1 .
\end{aligned}
$$

Then the zero solution of (9) is exponentially stable with respect to a ball $\Omega(R)$. Moreover, any solution $x(k)$ of (9) with initial condition $x_{0} \in \Omega(R)$ satisfies the inequality

$$
\sup _{k \geq 1}\|x(k)\| \leq \widehat{M}_{0}\left\|x_{0}\right\|(1-S(A(\cdot), f))^{-1},
$$

provided that

$$
\widehat{M}_{0}\left\|x_{0}\right\|(1-S(A(\cdot), f))^{-1}<R .
$$

Now, in order to apply Theorem 9 to solve the stability problem of the pseudolinear system (2), let us suppose that

$$
\begin{aligned}
& \rho_{0}(R)=\sup _{h \in \Omega(R), k \geq 0} \rho(\widehat{B}(h, k))<1, \\
& v_{0}(R)=\sup _{h \in \Omega(R), k \geq 0} g_{I}(\widehat{B}(h, k))<\infty .
\end{aligned}
$$

Put

$$
\tilde{\chi}(R)=\sup _{k \geq 1}\left(\sum_{j=0}^{k} \frac{k ! \rho_{0}^{k-j}(R) v_{0}^{j}(R)}{(k-j) !(j !)^{3 / 2}}\right) .
$$

Theorem 10. Under conditions (5), (6), and (38), assume that

(a) $\widehat{B}(z, k)(k=0,1, \ldots)$ are Hilbert-Schmidt operators satisfying condition (5), with $z \in \Omega(R)$,

(b)

$$
S(\widehat{B}(\cdot))=\sum_{k=0}^{\infty} \frac{v_{0}^{k}(R)}{\sqrt{k !}}\left[\frac{(k+1) q_{0}(R)}{\left(1-\rho_{0}(R)\right)^{k+2}}\right]<1 .
$$

Then the zero solution of (2) is exponentially stable with respect to a ball $\Omega(R)$. Moreover, any solution $x(k)$ of $(2)$ with initial condition $x_{0} \in \Omega(R)$ satisfies the inequality

$$
\sup _{k \geq 1}\|x(k)\| \leq \tilde{\chi}(R)\left\|x_{0}\right\|(1-S(\widehat{B}(\cdot)))^{-1},
$$

provided that

$$
\tilde{\chi}(R)\left\|x_{0}\right\|(1-S(\widehat{B}(\cdot)))^{-1}<R .
$$

Proof. By Theorem 8, we obtain

$$
\begin{array}{r}
\left\|(\widehat{B}(h, s))^{k}\right\| \leq \sum_{j=0}^{k} \frac{k !(\rho(\widehat{B}(h, s)))^{k-j} g_{I}^{j}(\widehat{B}(h, s))}{(k-j) !(j !)^{3 / 2}}, \\
h \in \Omega(R) ; k, s \geq 0 .
\end{array}
$$

This yields

$$
\begin{aligned}
& \sup _{h \in \Omega(R), s \geq 0}\left\|(\widehat{B}(h, s))^{k}\right\| \\
& \leq \sup _{h \in \Omega(R), s \geq 0}\left(\sum_{j=0}^{k} \frac{k !(\rho(\widehat{B}(h, s)))^{k-j} g_{I}^{j}(\widehat{B}(h, s))}{(k-j) !(j !)^{3 / 2}}\right) .
\end{aligned}
$$

Hence, by (38), we obtain

$$
p(R, k) \leq \sum_{j=0}^{k} \frac{k !\left(\rho_{0}(R)\right)^{k-j}\left(v_{0}(R)\right)^{j}}{(k-j) !(j !)^{3 / 2}}, \quad \forall k \geq 1 .
$$

It follows that

$$
\begin{aligned}
\chi(R) & =\sup _{k \geq 0} p(R, k) \\
& \leq \sup _{k \geq 1}\left(\sum_{j=0}^{k} \frac{k !\left(\rho_{0}(R)\right)^{k-j}\left(v_{0}(R)\right)^{j}}{(k-j) !(j !)^{3 / 2}}\right) .
\end{aligned}
$$

Proceeding in a similsr way, we obtain that

$$
\begin{aligned}
& q_{0}(R) \sum_{k=0}^{\infty} k p(R, k) \\
& \quad \leq \sum_{k=0}^{\infty} \frac{\left(v_{0}(R)\right)^{k}}{\sqrt{k !}}\left(\frac{(k+1) q_{0}(R)}{\left(1-\rho_{0}(R)\right)^{k+2}}\right) .
\end{aligned}
$$

Hence, from Theorem 9, the result follows.

Consider a perturbed system of autonomous pseudolinear equations

$$
\Delta x(k)=B(x(k)) x(k), \quad x(0)=x_{0} ; k=0,1, \ldots,
$$

where $B(z)$ is a linear and continuous operator in $\omega_{\infty}(R)$.

Let suppose that

$$
\begin{aligned}
& \tilde{\rho}_{0}(R)=\sup _{h \in \Omega(R)} \rho(\widehat{B}(h))<1, \\
& \widetilde{v}_{0}(R)=\sup _{h \in \Omega(R)} g_{I}(\widehat{B}(h))<\infty .
\end{aligned}
$$


Put

$$
\tilde{\chi}(R)=\sup _{k \geq 1}\left(\sum_{j=0}^{k} \frac{k ! \tilde{\rho}_{0}^{k-j}(R) \widetilde{v}_{0}^{j}(R)}{(k-j) !(j !)^{3 / 2}}\right)
$$

In this particular case $\mu(R)=0$ because system (48) is autonomous.

Corollary 11. Under conditions (5) and (49) assume that

(a) $\widehat{B}(z)$ is a Hilbert-Schmidt operator, with $z \in \Omega(R)$,

(b)

$$
\widetilde{S}(\widehat{B}(\cdot))=\gamma(R) M(R) \sum_{k=0}^{\infty} \frac{\widetilde{v}_{0}^{k}(R)}{\sqrt{k !}\left(1-\widetilde{\rho}_{0}(R)\right)^{k+2}}<1 .
$$

Then the zero solution of (48) is exponentilly stable. Moreover, any solution $x(k)$ of (48), with initial condition $x_{0} \in \Omega(R)$, satisfies

$$
\sup _{k \geq 1}\|x(k)\| \leq \tilde{\chi}(R)\left\|x_{0}\right\|(1-\widetilde{S}(\widehat{B}(\cdot)))^{-1}
$$

provided that

$$
\widetilde{\chi}(R)\left\|x_{0}\right\|(1-\widetilde{S}(\widehat{B}(\cdot)))^{-1}<R
$$

Proof. The leading part of (48), $B(x)$, is invariant with respect to the second argument; then $\mu(R)=0$. Besides, formulae (49) are independent of time. Consequently, this corollary is a consequence of Theorem 10 .

Example 12. We present an example that illustrates Theorem 10. In a finite-dimensional Hilbert space $\left(E^{2}, \|\right.$. $\left.\|_{\infty}\right)\left(E^{2}\right.$ the 2 -dimensional Euclidean space) and $\|x\|_{\infty}=$ $\max \left\{\left|x_{1}\right|,\left|x_{2}\right|\right\}$, with an appropriate inner product, we consider a system of difference equations

$$
\begin{aligned}
& \Delta\left(\begin{array}{c}
u(k) \\
v(k)
\end{array}\right) \\
& =\left(\begin{array}{cc}
0 & 1 \\
-\xi(u(k), v(k), k) & -f(u(k), v(k), k)
\end{array}\right)\left(\begin{array}{l}
u(k) \\
v(k)
\end{array}\right),
\end{aligned}
$$

assuming that the functions $f=f(u, v, k)$ and $\xi=\xi(u, v, k)$ are positive, bounded, and continuous with respect to the arguments $(u, v)$, under the conditions

$$
\begin{aligned}
k & \geq 0, \\
u^{2}+v^{2} & \leq R^{2}
\end{aligned}
$$

with a fixed positive number $R<\infty$. Thus,

$$
B((u(k), v(k)), k)=\left(b_{i j}(u, v, k)\right)_{i, j=1,2},
$$

where $b_{11}(u, v, k)=0, b_{12}(u, v, k)=1, b_{21}(u, v, k)=-\xi$, and $b_{22}(u, v, k)=-f$.
Hence, using the inequality

$$
g^{2}(A) \leq N^{2}(A)-\left|\operatorname{Trace} A^{2}\right|,
$$

the Hilbert-Schmidt norm of a matrix $A$, we obtain

$$
\widehat{v}_{0}(R)=2+\sup _{k \geq 0, u^{2}+v^{2} \leq R^{2}} \xi(u, v, k),
$$

provided that the supremum is finite. Consider

$$
\begin{gathered}
q_{0}(R)=\sup _{k \geq 0, u^{2}+v^{2} \leq R^{2}}\{|f(u, v, k+1)-f(u, v, k)|, \\
|\xi(u, v, k+1)-\xi(u, v, k)|\} \\
\rho_{0}(R)=\inf _{k \geq 0, u^{2}+v^{2} \leq R^{2}}\left\{\frac{f(u, v, k)}{2}\right. \\
\left.-\left(\frac{f(u, v, k)}{4}-\xi(u, v, k)\right)^{1 / 2}\right\} .
\end{gathered}
$$

If the inequality

$$
S(R)=\widetilde{q}_{0}(R)\left\{\frac{1}{\left(1-\rho_{0}(R)\right)^{2}}+\frac{2 \widehat{v}_{0}(R)}{\left(1-\rho_{0}(R)\right)^{3}}\right\}<1
$$

holds, then, by Theorem 10, the zero solution of (54) is exponentially stable with respect to the ball $u^{2}+v^{2} \leq R^{2}$. Moreover, any solution $x(k)=(u(k), v(k))$ of (54), with initial value $x(0)=(u(0), v(0)) \in E^{2}$, satisfies the inequality

$$
\sup _{k \geq 1}\|x(k)\| \leq \tilde{\chi}(R)\|x(0)\|(1-S(R))^{-1},
$$

provided that

$$
\tilde{\chi}(R)\|x(0)\|(1-S(R))^{-1}<R,
$$

where

$$
\tilde{\chi}(R)=\sup _{k \geq 1}\left\{\rho_{0}^{k}(R)+k \widehat{v}_{0}(R) \rho_{0}^{k-1}(R)\right\} .
$$

Example 13. Let $H=l_{2}(\mathbb{C})$, where $l_{2}:=l_{2}(\mathbb{C})$ is the Hilbert space of complex sequences, equipped with the norm

$$
\|u\|_{l_{2}}=\left(\sum_{j=1}^{\infty}\left|u_{j}\right|^{2}\right)^{1 / 2}, \quad u=\left(u_{j}\right) \in l_{2} .
$$

Consider the infinite-dimensional triangular system, defined on $l_{2}$ :

$$
\Delta x(k)=B(x(k)) x(k), \quad x(0)=x_{0} \in \Omega(R),
$$

where $B(z)$ is a linear and continuous operator, represented by the infinite-dimensional matrix

$$
B(z)=\left(b_{i j}(z)\right), \quad 1 \leq i \leq j ; z \in l_{2} .
$$


Additionally, assume that $B(z)$ satisfies the following conditions:

(a) There exists a nonnegative $\gamma(R)$ such that

$$
\left\|\left(B\left(h_{1}\right)-B(h)\right) \omega\right\| \leq \gamma(R)\left\|h_{1}-h\right\|\|\omega\|,
$$

where $\gamma(R)$ is independent of $\omega \in \Omega(R)$.

(b) $\widetilde{\rho}_{0}(R)=\sup _{h \in \Omega(R)}\left\{e^{b_{i i}(h)}\right\}<1, i=1,2, \ldots$.

(c) $\widetilde{v}_{0}(R) \leq J(\widehat{B}(\cdot))<\infty$, where

$J(\widehat{B}(\cdot))$

$=\frac{1}{\sqrt{2}} \sup _{h \in \Omega(R)}\left(\sum_{j=1}^{\infty}\left[\sum_{k=1, k \neq j}^{\infty}\left|b_{j k}(h)-\bar{b}_{j k}(h)\right|^{2}\right]\right)^{1 / 2}$

(d) $\tilde{\chi}(R) \leq \widetilde{\chi}_{1}(R)$, where

$$
\tilde{\chi}_{1}(R)=\sup _{k \geq 1}\left(\sum_{j=0}^{k} \frac{\left(\widetilde{\rho}_{0}(R)\right)^{k-j} J^{j}(\widehat{B}(\cdot))}{(k-j) !(j !)^{3 / 2}}\right) .
$$

(e)

$$
\widetilde{S}_{1}(\widehat{B}(\cdot))=\gamma(R) M(R) \sum_{k=0}^{\infty} \frac{J^{k}(\widehat{B}(\cdot))}{\sqrt{k !}\left(1-\widetilde{\rho}_{0}(R)\right)^{k+2}}<1 .
$$

Then the zero solution of (65) is exponentially stable. Moreover, any solution $x(k)$ of (65), with the initial condition $x_{0} \in$ $\Omega(R)$, satisfies the inequality

$$
\sup _{k \geq 1}\|x(k)\| \leq \tilde{\chi}_{1}(R)\left\|x_{0}\right\|\left(1-\widetilde{S}_{1}(\widehat{B}(\cdot))\right)^{-1},
$$

provided that

$$
\widetilde{\chi}_{1}(R)\left\|x_{0}\right\|\left(1-\widetilde{S}_{1}(\widehat{B}(\cdot))\right)^{-1}<R \text {. }
$$

In fact,

$$
\begin{aligned}
\tilde{\rho}_{0}(R) & =\sup _{h \in \Omega(R)} \rho(\widehat{B}(\cdot)) \\
& =\sup _{h \in \Omega(R)}\left\{1+b_{i i}(h)\right\}, \quad i=1,2, \ldots \\
& \leq \sup _{h \in \Omega(R)}\left\{e^{b_{i i}(h)}\right\}, \quad i=1,2, \ldots
\end{aligned}
$$

In many cases, $g_{I}(A)$ is difficult to calculate. But using the inequality

$$
g_{I}^{2}(A) \leq \frac{1}{2} N^{2}\left(A_{I}\right)
$$

we obtain a new estimation for $g_{I}(A)$; namely,

$$
g_{I}(A) \leq \frac{1}{\sqrt{2}} \sum_{j=1}^{\infty}\left(\sum_{k=1, k \neq j}^{\infty}\left|a_{j k}-\bar{a}_{j k}\right|^{2}\right)^{1 / 2} .
$$

It follows that

$$
\begin{aligned}
\widetilde{v}_{0}(R) & =\sup _{h \in \Omega(R)} g_{I}(\widehat{B}(h)) \\
& \leq \frac{1}{\sqrt{2}} \sup _{h \in \Omega(R)} \sum_{j=1}^{\infty}\left(\sum_{k=1, k \neq j}^{\infty}\left|b_{j k}(h)-\bar{b}_{j k}(h)\right|^{2}\right)^{1 / 2} \\
& :=J(\widehat{B}(\cdot))<\infty .
\end{aligned}
$$

Consequently, $\widehat{B}(h)$ is a Hilbert-Schmidt operator for $h \epsilon$ $\Omega(R)$. On the other hand,

$$
\begin{aligned}
\tilde{\chi}(R) & =\sup _{k \geq 1}\left(\sum_{j=0}^{\infty} \frac{\left(\widetilde{\rho}_{0}(R)\right)^{k-j}\left(\widetilde{v}_{0}(R)\right)^{j}}{(k-j) !(j !)^{3 / 2}}\right) \\
& \leq \sup _{k \geq 1}\left(\sum_{j=0}^{\infty} \frac{\left(\widetilde{\rho}_{0}(R)\right)^{k-j} J^{j}(\widehat{B}(\cdot))}{(k-j) !(j !)^{3 / 2}}\right):=\widetilde{\chi}_{1}(R) .
\end{aligned}
$$

Finally,

$$
\begin{aligned}
\widetilde{S}(\widehat{B}(\cdot)) & =\gamma(R) M(R) \sum_{k=0}^{\infty} \frac{\left(\widetilde{v}_{0}(R)\right)^{k}}{\sqrt{k !}\left(1-\widetilde{\rho}_{0}(R)\right)^{k+2}} \\
& \leq \gamma(R) M(R) \sum_{k=0}^{\infty} \frac{J^{k}(\widehat{B}(\cdot))}{\sqrt{k !}\left(1-\widetilde{\rho}_{0}(R)\right)^{k+2}} .
\end{aligned}
$$

Then, by Corollary 11, the zero solution of (65) is exponentially stable. Moreover, any solution $x(k)$ of (65), with initial condition $x_{0} \in \Omega(R)$, satisfies the inequality

$$
\sup _{k \geq 1}\|x(k)\| \leq \widetilde{\chi}_{1}(R)\left\|x_{0}\right\|\left(1-\widetilde{S}_{1}(\widehat{B}(\cdot))\right)^{-1}
$$

provided that

$$
\widetilde{\chi}_{1}(R)\left\|x_{0}\right\|\left(1-\widetilde{S}_{1}(\widehat{B}(\cdot))\right)^{-1}<R .
$$

\section{Conclusions}

New conditions for the exponential stability of a class of infinite-dimensional nonlinear difference systems are derived. We establish the robustness of the exponential stability, in the sense that the exponential stability for a given pseudolinear equation persists under sufficiently small perturbations. Unlike the classic method of stability analysis, we do not use the technique of Lyapunov function in the process of construction of the stability results. The proofs are carried out using the semigroup theory combined with the freezing method. That is, the equation is represented as a perturbation about a fixed value of the operator and then applying norm estimates for operator-valued functions the results follow. We have presented two examples which show how this approach brings out different aspects of the stability problem of pseudolinear equations. 


\section{Competing Interests}

The author declares that there are no competing interests regarding the publication of this paper.

\section{Acknowledgments}

This research was supported by Fondecyt Chile under Grant no. 1130112.

\section{References}

[1] R. P. Agarwal, H. B. Thompson, and C. C. Tisdell, "Difference equations in Banach spaces," Computers \& Mathematics with Applications, vol. 45, no. 6-9, pp. 1437-1444, 2003.

[2] M. I. Gil' and R. Medina, "The freezing method for linear difference equations," Journal of Difference Equations and Applications, vol. 8, no. 5, pp. 485-494, 2002.

[3] S. M. Lee and J. H. Park, "Robust stabilization of discretetime nonlinear Lur'e systems with sector and slope restricted nonlinearities," Applied Mathematics and Computation, vol. 200, no. 1, pp. 429-436, 2008.

[4] R. Memarbashi, "Sufficient conditions for the exponential stability of non-autonomous difference equations," Applied Mathematics Letters, vol. 21, no. 3, pp. 232-235, 2008.

[5] R. Medina, "Exponential stabilization of nonlinear discretetime systems," Journal of Difference Equations and Applications, vol. 17, no. 5, pp. 697-708, 2011.

[6] B. Sasu and A. L. Sasu, "Stability and stabilizability for linear systems of difference equations," Journal of Difference Equations and Applications, vol. 10, no. 12, pp. 1085-1105, 2004.

[7] T.-L. Hsien and C.-H. Lee, "Exponential stability of discrete time uncertain systems with time-varying delay," Journal of the Franklin Institute, vol. 332, no. 4, pp. 479-489, 1995.

[8] N. S. Bay and V. N. Phat, "Stability analysis of nonlinear retarded difference equations in Banach spaces," Computers \& Mathematics with Applications, vol. 45, no. 6-9, pp. 951-960, 2003.

[9] R. Medina and M. I. Gil, "The freezing method for abstract nonlinear difference equations," Journal of Mathematical Analysis and Applications, vol. 330, no. 1, pp. 195-206, 2007.

[10] P. H. A. Ngoc and L. T. Hieu, "New criteria for exponential stability of nonlinear difference systems with time-varying delay," International Journal of Control, vol. 86, no. 9, pp. 16461651, 2013.

[11] J. Peuteman and D. Aeyels, "Averaging results and the study of uniform asymptotic stability of homogeneous differential equations that are not fast time-varying," SIAM Journal on Control and Optimization, vol. 37, no. 4, pp. 997-1010, 1999.

[12] V. Solo, "On the stability of slowly time-varying linear systems," Mathematics of Control, Signals, and Systems, vol. 7, no. 4, pp. 331-350, 1994.

[13] V. Sundarapandian, "Exponential stabilizability and robustness analysis for discrete-time nonlinear systems," Applied Mathematics Letters. An International Journal of Rapid Publication, vol. 18, no. 7, pp. 757-764, 2005.

[14] J. Wang and X. Li, "Improved global exponential stability for delay difference equations with impulses," Applied Mathematics and Computation, vol. 217, no. 5, pp. 1933-1938, 2010.
[15] S. P. Banks, A. Moser, and D. Mccaffrey, "Robust exponential stability and evolution equations," Archives of Control Sciences, vol. 4, no. 3-4, pp. 261-279, 1995.

[16] A. I. Dvirnyi and V. I. Slyn'ko, "On stability of solutions of nonlinear nonstationary systems of impulsive differential equations in a critical case," Nonlinear Oscillations, vol. 14, no. 4, pp. 472-496, 2012.

[17] A. I. Dvirnyi and V. I. Slyn'ko, "Global stability of solutions of nonstationary monotone differential equations with impulsive action in the pseudolinear form," Nonlinear Oscillations, vol. 14, no. 2, pp. 193-210, 2011.

[18] K. S. Hong, J. W. Wu, and K.-I. Lee, "New conditions for the exponential stability of evolution equations," IEEE Transactions on Automatic Control, vol. 39, no. 7, pp. 1432-1436, 1994.

[19] A. A. Martynyuk, "Novel bounds for solutions of nonlinear differential equations," Applied Mathematics, vol. 6, no. 1, pp. 182-194, 2015.

[20] R. Medina, "Exponential stability of slowly varying discrete systems with multiple state delays," International Journal of Robust and Nonlinear Control, vol. 23, no. 13, pp. 1496-1509, 2013.

[21] B. F. Bylov, B. M. Grobman, V. V. Nemickii, and R. E. Vinograd, Theory of Lyapunov Exponents, Nauka, Moscow, Russia, 1966 (Russian).

[22] M. I. Gil', Norm Estimates for Operator-Valued Functions and Applications, Marcel Dekker, New York, NY, USA, 1995.

[23] R. Medina and C. Martinez, "Exponential stability criteria for discrete time-delay systems," International Journal of Robust and Nonlinear Control, vol. 25, no. 4, pp. 527-541, 2015. 


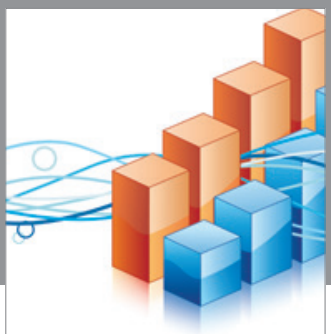

Advances in

Operations Research

vatem alat4

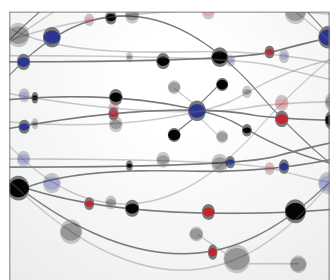

\section{The Scientific} World Journal
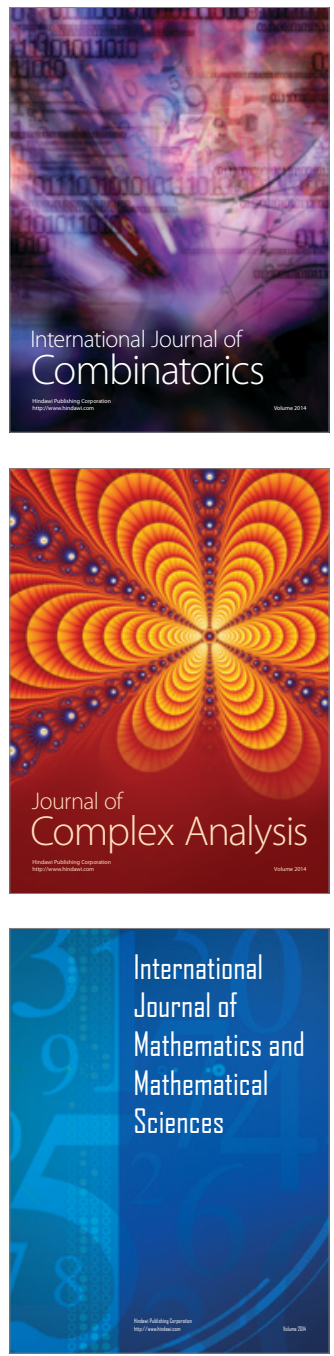
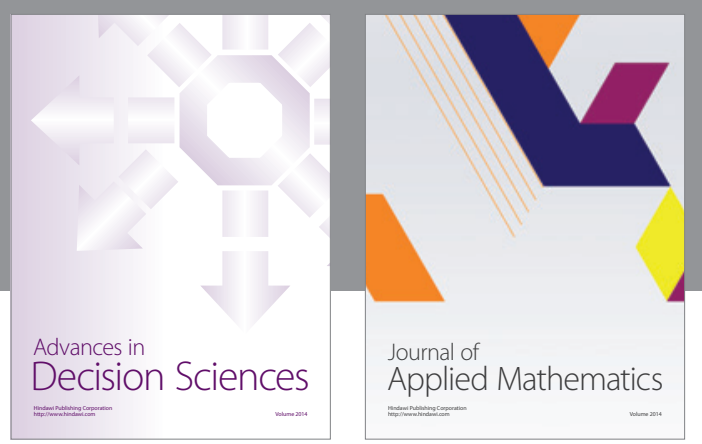

Algebra

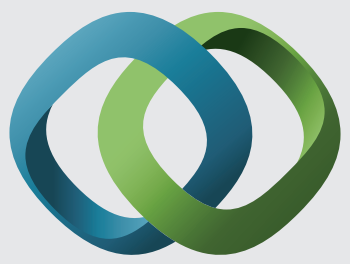

\section{Hindawi}

Submit your manuscripts at

http://www.hindawi.com
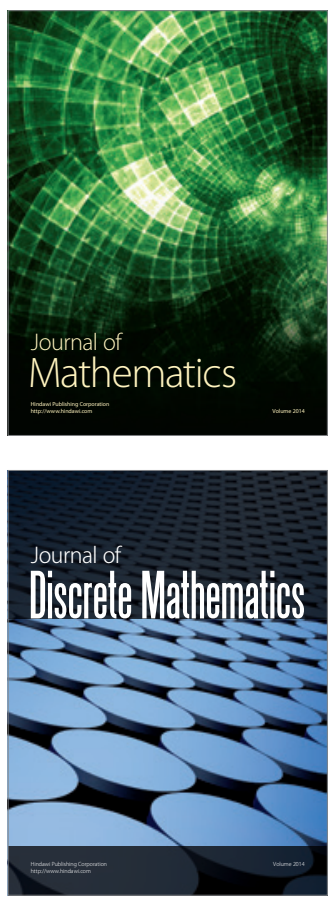

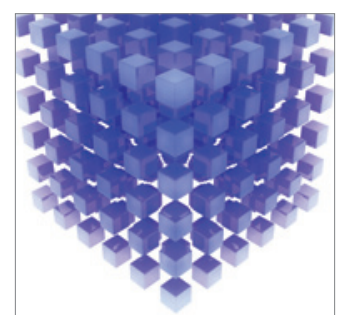

Mathematical Problems in Engineering
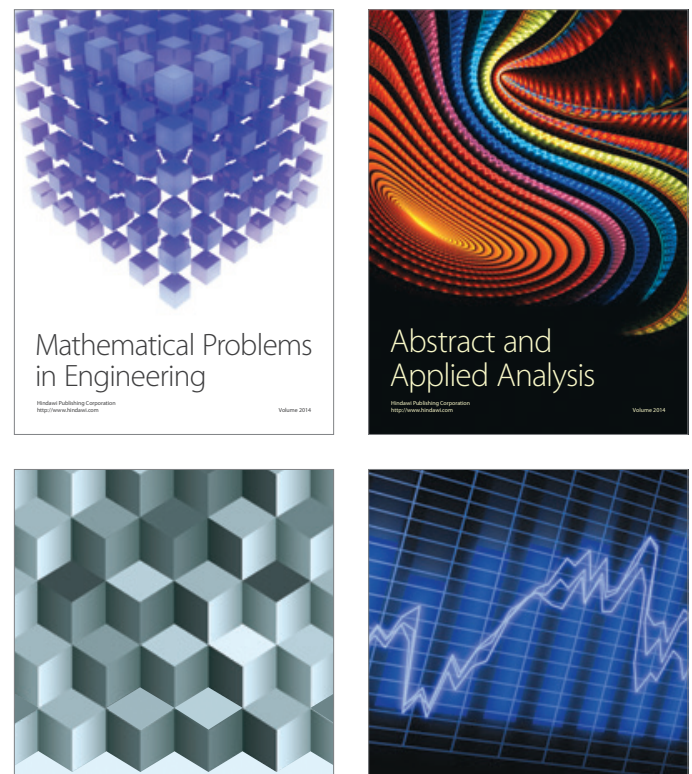

Journal of

Function Spaces

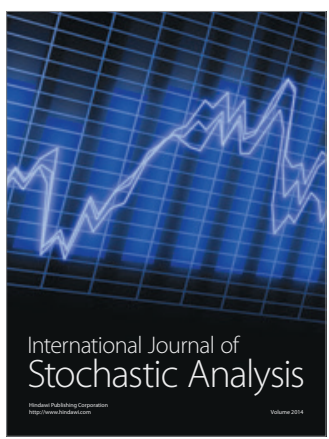

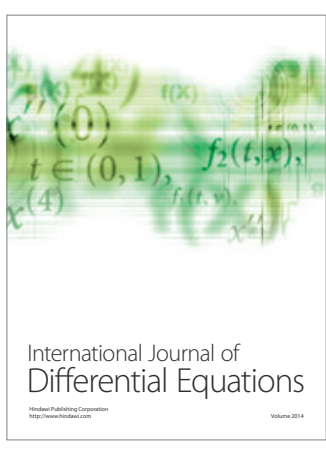
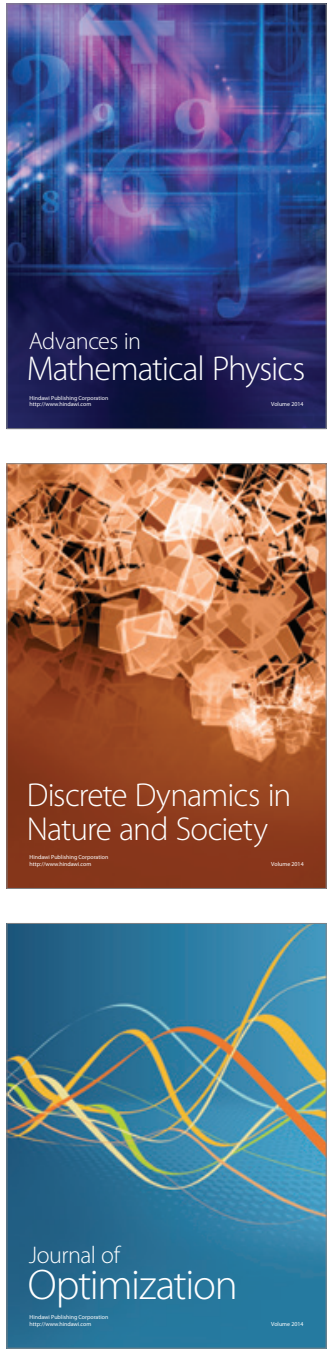